\title{
Das neugeborene Fohlen als Kolikpatient
}

\author{
Sophia Ennen
}

Zeigen Fohlen innerhalb der ersten Lebenstage eine Koliksymptomatik, ist rasches Handeln gefordert. Eine Vielzahl möglicher Ursachen kommt infrage und erst die korrekte Diagnosestellung ermöglicht die Einleitung der richtigen Therapie.

Fohlen, bei denen vor Ort keine abschlie-

ßende Diagnose gestellt werden kann, sollten in Absprache mit dem Halter möglichst frühzeitig in eine gut ausgestattete Klinik überwiesen werden. So geht keine Zeit verloren, falls chirurgisches oder intensivmedizinisches Eingreifen notwendig ist.

Einige Punkte sind im Gegensatz zum ausgewachsenen Kolik-Patienten zu beachten:

- Bei equinen Neonaten kommt es häufiger als bei adulten Pferden vor, dass eine Kolik nicht gastrointestinal bedingt ist.

- Angeborene Missbildungen sind als Auslöser des Krankheitsbilds in Betracht zu ziehen.

- Die Möglichkeit der transrektalen Untersuchung entfällt. Stattdessen ist nur eine digitale rektale Untersuchung möglich. Im Gegenzug dazu ist die Sonografie sehr viel leichter und vollständiger durchführbar als beim ausgewachsenen Pferd. In manchen Fällen sind auch abdominale Röntgenaufnah- men zur Diagnose der Kolikursache aufschlussreich.

- Der Einsatz von Butylscopolamin (als Bestandteil von Buscopan compositum, Fa. Boehringer Ingelheim) ist in der Koliktherapie bei equinen Neonaten in aller Regel verzichtbar. Stattdessen sollte bei der Erstbehandlung zunächst Metamizol als Monosubstanz Anwendung finden.

Die Auflistung möglicher Kolikursachen in diesem Artikel erhebt keinen Anspruch auf Vollständigkeit, und beschränkt sich auf die häufigsten sowie einige seltene Kolikursachen in den ersten Lebenstagen eines Pferdes.

\section{Diagnostik}

Das Symptom „Kolikschmerz“ ist bei neugeborenen Fohlen eindeutig festzustellen. Meist melden sich die Halter mit dem Vorbericht, das Fohlen wälze sich immer wieder und bleibe teilweise auch auf dem Rücken liegen ( $\triangleright$ Abb. 1). Häufig zeigen die Fohlen zusätzlich Tenesmus. Dabei ist es durch Adspektion nicht immer möglich zu unterscheiden, ob die Tiere auf Kot oder Harn drängen.

Aller Erfahrung nach pressen Fohlen mit gastrointestinalen Problemen bei einer

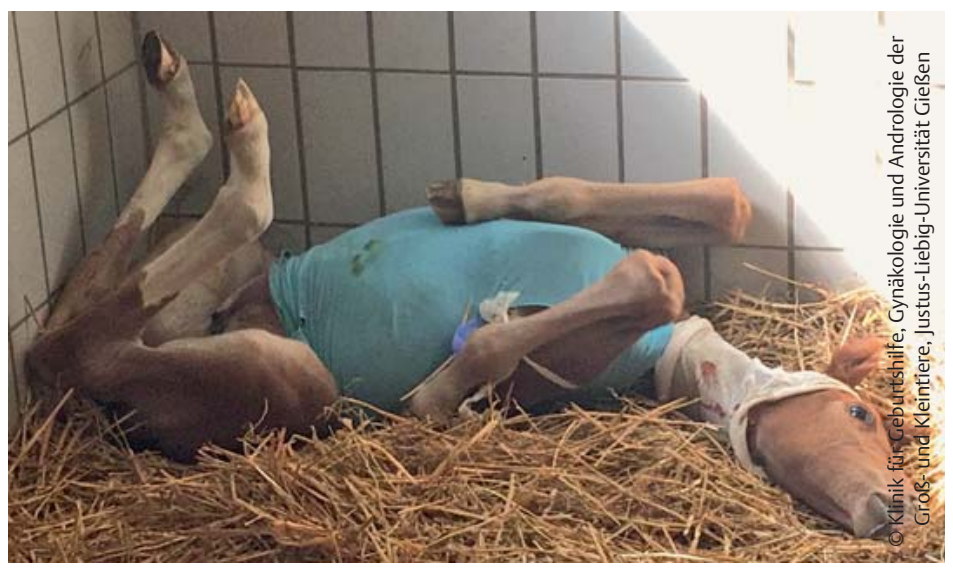

Abb. 1 Fohlen mit Kolik, das in Rückenlage verharrt.

Mekoniumverhaltung in kyphotischer Haltung, während Fohlen mit urologischen Problemen, wie beispielsweise einem Uroperitoneum, eher eine Lordose zeigen $(\bullet$ Abb. 2$)$.

\section{Vorbericht}

Wie bei jeder tierärztlichen Untersuchung spielt die möglichst vollständige Erhebung des Vorberichts eine große Rolle.

An dieser Stelle sollen einzelne Punkte aufgegriffen werden, die im Hinblick auf das Kolikgeschehen von Bedeutung sein können:

- Wie waren Gestationsdauer und Entwicklungszustand des Neonaten bei der Geburt?

Eines der vielen Probleme prämaturer Fohlen ist die Unreife des Darmes: Es treten Motilitäts- und Durchblutungsstörungen auf, die häufig erst einige Tage nach der Geburt klinisch in Erscheinung treten und eine Koliksymptomatik bedingen können.

- Was waren die Routinemaßnahmen zur Erstversorgung nach der Geburt? Neben der gegebenenfalls bereits erfolgten Verabreichung von Klistieren sind auch der Abnabelungsprozess und die Nabeldesinfektion zu erfragen, da Entzündungen des Nabels in Ausnahmefällen abdominale Schmerzen verursachen können. Außerdem ist die Vitamin-E-Selen-Versorgung anzusprechen, da die nutritive Muskeldystrophie bereits perinatal so ausgeprägt vorkommen kann, dass eine Saug- bzw. Trinkschwäche mit anschließender Hypogammaglobulinämie und Mekoniumobstipation resultiert. Stark bemuskelte Rassen (beispielsweise Quarter Horses, Pura Raza Española u. Ä.) sind von Muskeldystrophien besonders betroffen.

- Zu welchem Zeitpunkt nach der Geburt traten die Koliksymptome erstmalig auf und wie äußerten sich diese? 


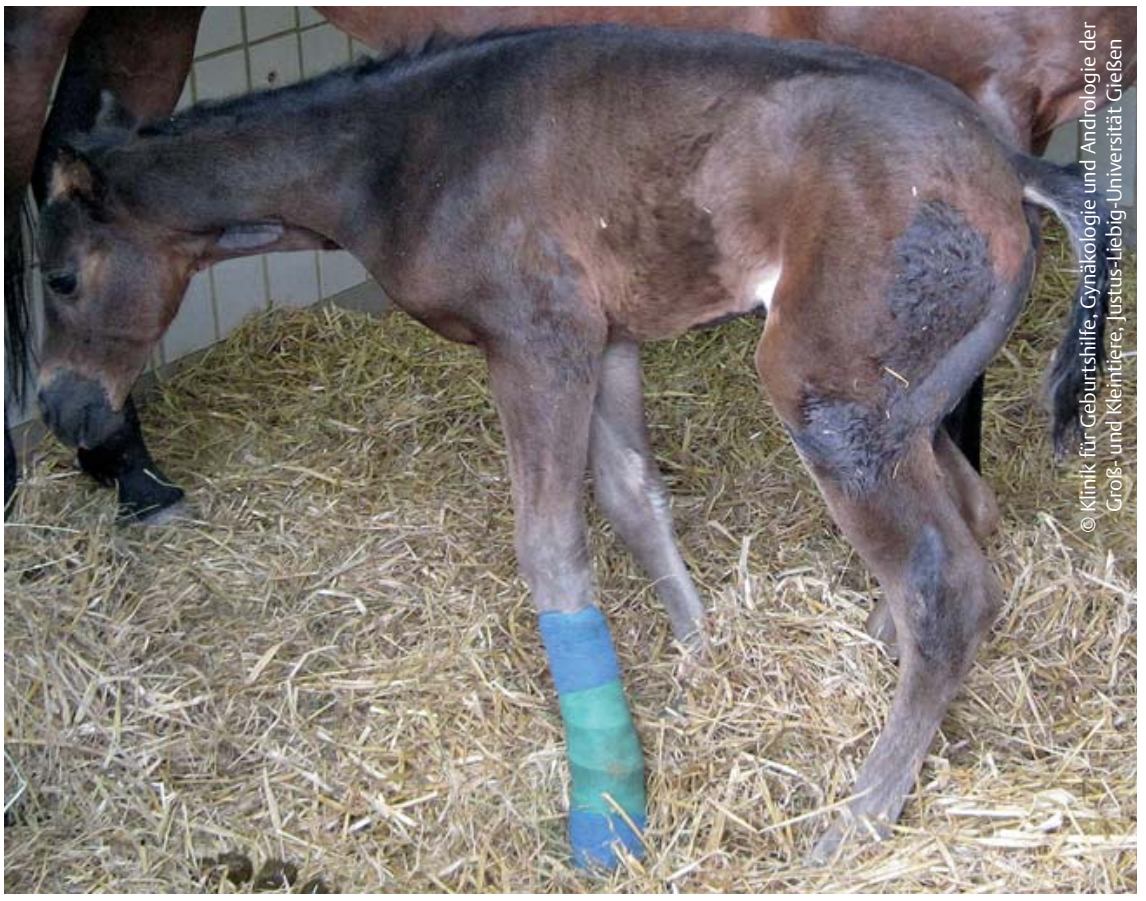

Abb. 2 Fohlen mit Tenesmus, das in kyphotischer Haltung presst.

Das Alter des Fohlens beim ersten Auftreten der Kolik spielt eine Rolle, aber auch, ob die Koliksymptomatik permanent oder anfallsartig auftritt oder eine zeitliche Verbindung zur Nahrungsaufnahme erkennbar ist. Vorsicht ist geboten, wenn ein vormals hochgradig kolikendes Fohlen plötzlich keine Schmerzen mehr zu haben scheint, aber stattdessen apathisch wirkt: Häufig liegt bei diesen Patienten eine Ruptur vor.

- Ist Mekonium oder bereits Milchkot abgegangen?

Die Gesamtlänge der aneinandergereihten Mekoniumballen sollte bei einem Warmblutfohlen $80-100 \mathrm{~cm}$ betragen [1]. Die Beobachtung, dass bereits Darmpech abgegangen ist, darf somit nicht mit der Tatsache gleichgesetzt werden, dass keine Mekoniumverhaltung vorliegt.

- Gab oder gibt es Besonderheiten beim Harnabsatz?

In den ersten 8 Lebensstunden sollte mindestens 1-mal eine größere Menge Harn im Strahl abgesetzt worden sein. Ständiges Pressen und Harnträufeln sind nicht physiologisch. Bei Hengstfohlen kommt es vor, dass sie im Laufe der ersten Lebenstage während des Harnabsatzes nicht ausschachten. Diese Verhaltensweise stellt keinen Grund zur Besorgnis dar, solange ausreichen- de Mengen an Harn abgesetzt werden. Möglicherweise hat der Besitzer auch schon ein Harnträufeln aus dem Nabel beim Harnabsatz beobachtet, was auf eine Urachusfistel hindeuten würde.

- Welche Behandlungsversuche sind bereits erfolgt?

Von besonderem Interesse ist diesbezüglich die Verabreichung von nichtsteroidalen Antiphlogistika (NSAID), und zwar zum einen, damit die Schmerzäußerungen des Fohlens bei der Untersuchung realistisch eingeschätzt werden können. Zum anderen ist eine mögliche Vorbehandlung von Fohlen und Mutterstute mit NSAID von Interesse, da bei längerer Vorbehandlung Ulzerationen des Magens bestehen können oder zukünftig zu erwarten sind, wenn keine entsprechende Prophylaxe erfolgt.

Bei Mekoniumobstipationen sollte abhängig vom Naturell des Besitzers erfragt werden, ob im Vorfeld Therapieversuche mit mehr oder weniger geeigneten Geräten und Spüllösungen erfolgt sind.

\section{Grunduntersuchung des Fohlens}

Auch wenn die Ursache für das Symptom „Kolik“ mit hoher Wahrscheinlichkeit im
Bereich des Abdomens zu finden ist, führt kein Weg an einer sorgfältigen segmentalen Untersuchung vorbei [2].

Diese gründliche Untersuchung „von Kopf bis Fuß“ ist unter anderem aus dem Grund unumgänglich, dass neugeborene Patienten angeborene Missbildungen aufweisen können. Wird ein Fohlen mit einer akuten Koliksymptomatik in eine Klinik überwiesen und dort bei der Aufnahmeuntersuchung außer der eventuell zu therapierenden Kolikursache beispielsweise eine hochgradige Gaumenspalte festgestellt, wird diese zuvor noch nicht gestellte Diagnose in aller Regel zur Euthanasie des Fohlens führen. Solche und ähnliche Szenarien sind in hohem Maße ärgerlich für die Besitzer und durch eine vollständige Erstuntersuchung vermeidbar.

Außerdem bedingen sich beim Fohlen zahlreiche Krankheitszustände gegenseitig. So sollte beispielsweise die Diagnose „Mekoniumobstipation“ nicht dazu führen, nur diese zu therapieren und andere Erkrankungen außer Acht zu lassen: Unzureichender Mekoniumabsatz ist häufig durch eine mangelhafte Kolostrumversorgung verursacht.

Bei allen Fohlen mit einer Mekoniumobstipation - auch wenn diese konservativ therapierbar ist - sollte der IgGGehalt des Blutes untersucht werden, um eine Hypogammaglobulinämie und eine damit bevorstehende Sepsis frühzeitig festzustellen.

Einige Kolikursachen können bereits durch die klinische Grunduntersuchung festgestellt werden, beispielsweise:

- Darmpechverhaltung

- Atresia ani

- inkarzerierte Hernien an Nabel und Leiste

Außerdem kann der Füllungszustand des Abdomens wichtige Hinweise in Bezug auf die Genese liefern.

\section{Weiterführende Untersuchungen}

Steht die Diagnose nach Abschluss der Grunduntersuchung noch aus, sind weiterführende Untersuchungen nötig. Unter 
Tab. 1 Häufige Ursachen für das Auftreten einer Koliksymptomatik bei neugeborenen Fohlen (Auswahl).

\begin{tabular}{|l|l|}
\hline Kolikursache & Diagnostik durch \\
\hline Mekoniumobstipation & digitale rektale Untersuchung, Sonografie \\
\hline Uroperitoneum & $\begin{array}{l}\text { Palpation, Sonografie (Abdominozentese/ } \\
\text { Labordiagnostik) }\end{array}$ \\
\hline (bevorstehende) Enteritis bzw. Enterotoxämie & $\begin{array}{l}\text { Auskultation, Kotuntersuchung, Elektrolyte, } \\
\text { Säure-Basen-Status (Sonografie) }\end{array}$ \\
\hline Ileus/Obstruktionen & Sonografie, (Probe-)Laparotomie \\
\hline Proktitis & $\begin{array}{l}\text { Anamnese, Adspektion, Ausschluss anderer } \\
\text { Ursachen }\end{array}$ \\
\hline $\begin{array}{l}\text { Motilitätsstörungen (eher selten, aber häufig } \\
\text { bei prämaturen Fohlen) }\end{array}$ & $\begin{array}{l}\text { Auskultation, Sonografie: Kolik v. a. } \\
\text { postprandial }\end{array}$ \\
\hline $\begin{array}{l}\text { Magenulzera - Fohlen in den ersten Lebens- } \\
\text { tagen sind eher selten betroffen, danach sind } \\
\text { Ulzerationen aber eine häufige Kolikursache }\end{array}$ & $\begin{array}{l}\text { Vorbericht, klinische Hinweise (Bruxismus/ } \\
\text { Salivation), okkultes Blut im Kot, Gastroskopie }\end{array}$ \\
\hline
\end{tabular}

diesen nimmt die Sonografie einen besonderen Stellenwert ein ( $\vee$ Tab. 1). Außerdem kommen Labordiagnostik (Blut, Kot, Urin), weitere bildgebende Verfahren und die Abdominozentese infrage.

\section{Sonografie}

Die sonografische Untersuchung des Fohlens lässt sich auch im Heimatbestand ohne großen Aufwand durchführen. Abhängig von Temperament und Größe des Fohlens kann die Untersuchung am stehenden oder liegenden Patienten erfolgen.

Die Untersuchung am stehenden Patienten bietet den Vorteil, dass flüssigkeitsgefüllte Darmsegmente sowie intraperitoneale Flüssigkeit nach ventral absinken und so leichter auffindbar sind [3]. Unabhängig von der Lagerung des Fohlens sollten mindestens 2 Hilfspersonen zur Verfügung stehen. Bei Untersuchungen mit nur 1 Hilfsperson kann alternativ auch eine Sedation erfolgen. Dafür bietet sich beispielsweise die langsame intravenöse Verabreichung von Diazepam in einer Dosierung von $0,1 \mathrm{mg} / \mathrm{kg}$ Körpergewicht an.

Eine $\mathbf{5} \mathbf{~ M H z}-$ Rektalsonde, wie sie in aller Regel für die gynäkologische Untersuchung von Stuten eingesetzt wird, ist in vielen Fällen ausreichend. Der Einsatz von 7,5-10 MHz-Schallköpfen (Linear- oder Konvexscanner) führt allerdings zu qualitativ hochwertigeren Bildern, die leichter auswertbar sind [4].
Eine Rasur ist bei den meisten Fohlen nicht nötig, kann aber bei Tieren mit dichtem Fell die Detailerkennbarkeit verbessern. Soll das Fell verbleiben, ist das großzügige Aufbringen von Alkohol angebracht, was je nach Untersuchungsdauer regelmäßig zu wiederholen ist.

Bei der sonografischen Untersuchung sollte jeder Untersucher sein eigenes Schema entwickeln, um das gesamte Abdomen beurteilen zu können.

Während das Erkennen von freier Flüssigkeit recht einfach ist, braucht es für die $\mathrm{Er}$ hebung anderer Befunde, wie beispielsweise Veränderungen der Darmmotilität, weitaus mehr Erfahrung. Ein gesunder Dünndarm kontrahiert sich pro Minute durchschnittlich 2- bis 2,5-mal zirkulär, sodass dieser Wert zur Objektivierung herangezogen werden kann. Zusätzlich sollte die Darmwanddicke ausgemessen werden: Unabhängig vom Darmsegment sollte sie nicht mehr als $3 \mathrm{~mm}$ betragen, andernfalls ist von entzündlichen oder ödematösen Veränderungen auszugehen [3].

\section{Übersicht über einzelne Kolikursachen}

\section{Mekoniumobstipation}

Fohlen mit Mekoniumobstipation werden - je nach Schwere des Falles - meist an ihrem ersten, aber so gut wie immer in- nerhalb der ersten 3 Lebenstage vorgestellt. Häufig tritt die Erkrankung bei lebensschwachen Fohlen oder Tieren mit schlechtem Saugreflex auf, weil in diesen Fällen die laxierende Wirkung des Kolostrums fehlt. Außerdem kann die Erkrankung durch eine kongenitale Enge des knöchernen Beckens bedingt sein; von dieser Form sind häufig Vollblut-Hengstfohlen betroffen. Das Becken solcher Tiere kann so eng sein, dass selbst eine digitale rektale Untersuchung mit reichlich Gleitgel misslingt und somit auch größere Mekoniumballen nicht durchtreten können.

Ist bei der digitalen rektalen Untersuchung kein Mekonium zu erreichen, schließt das ein Darmpechverhalten nicht zwangsläufig aus.

Gerade bei großrahmigen Fohlen gelangt der untersuchende Finger nicht an das kraniale Ende des Beckens, sodass dieser „Negativ-Befund“ in jedem Fall durch die Sonografie zu ergänzen ist. Das Gleiche gilt auch für das Absetzen von Milchkot in geringen Mengen. Liegen nur noch 2 oder 3 größere Mekoniumballen direkt kranial des Beckens, können kleine Portionen von Milchkot durch starkes Pressen am Darmpech vorbeigeschoben werden.

Das sonografische Bild ist eindeutig: Kranial und/oder dorsal der Blase lassen sich die hyperechogenen Mekoniumballen im Colon descendens zweifelsfrei darstellen. Besteht die Möglichkeit, ein abdominales Röntgenbild anzufertigen (latero-lateral), so lassen sich die Mekoniumballen und eine eventuell davorliegende Aufgasung ebenfalls gut darstellen.

\section{Uroperitoneum}

Bei einem Uroperitoneum ist meist die Harnblase rupturiert, in selteneren Fällen liegen eine innere Urachusfistel, eine Hemmungsmissbildung der Blase oder eine Missbildung beziehungsweise Verletzung der Ureteren vor. Letztere kann beispielsweise durch eine weit dorsal gelegene Rippenfraktur hervorgerufen werden.

Je nach Größe des Defekts werden die Fohlen nach 2-10 Tagen vorgestellt. Häufig ist Trinkschwäche das erste Symptom. Ist die Läsion sehr klein, kann anfangs noch Harn im Strahl abgesetzt werden. In weiter fort- 

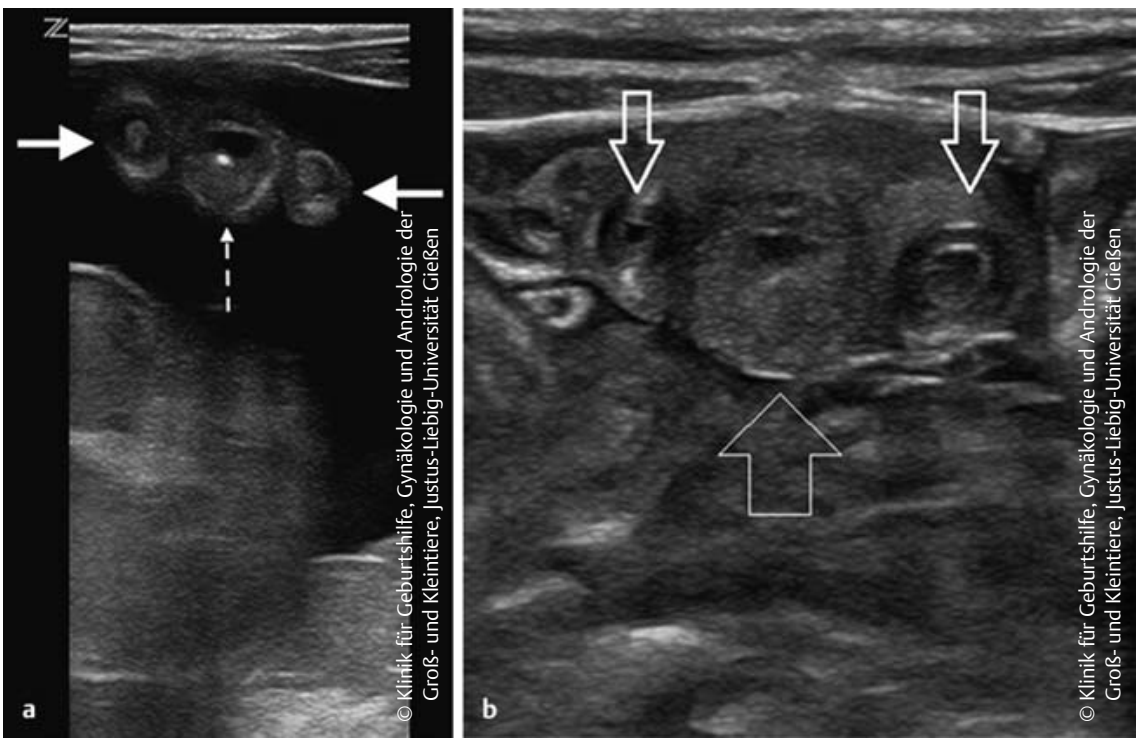

Abb. $\mathbf{3}$ a und $\mathbf{b}$ a Fohlen mit Uroperitoneum. Es sind große Mengen anechogene Flüssigkeit (schwarz) zu erkennen. Die dicken Pfeile kennzeichnen die beiden Nabelarterien. Zentral (gestrichelter Pfeil) ist der Übergang vom Urachus zum kranialen Harnblasenpol erkennbar. b Fohlen ohne Uroperitoneum, bei dem die gleichen Strukturen wie in > Abb. $\mathbf{3}$ a dargestellt werden können: Die beiden Nabelarterien (dicke Pfeile) flankieren den Übergang vom Urachus zum kranialen Harnblasenpol. Wenn pathologische Mengen freier Flüssigkeit fehlen, liegen die Nabelstrukturen der Bauchwand direkt an.

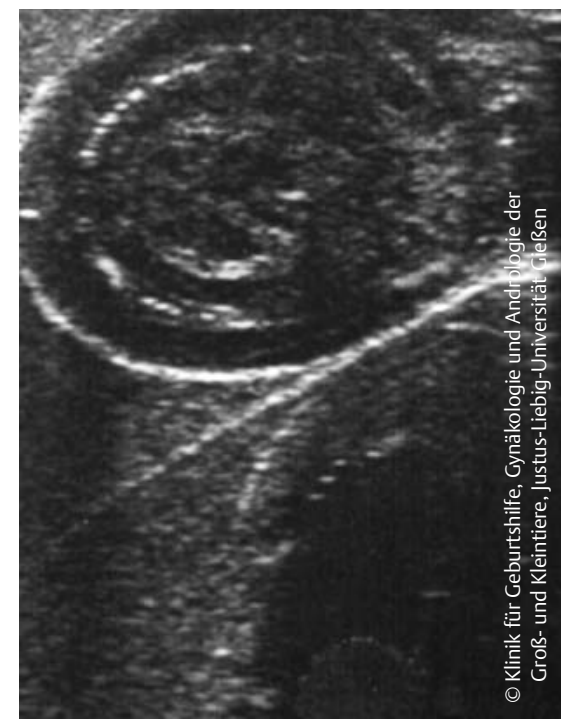

Abb. 4 Sonografischer Querschnitt durch eine Invagination des Dünndarms. Die ineinander geschichteten, ödematös verdickten Darmwände sind deutlich zu erkennen.

Tab. 2 Kalium- und Natriumwerte von einem Quarter-Horse-Fohlen, das am 7. Lebenstag mit der Diagnose Uroperitoneum vorgestellt wurde.

\begin{tabular}{|lllc|}
\hline Parameter & vor Infusion & $\begin{array}{l}\text { nach Infusion von } \\
1 \text { Liter } \mathrm{NaCl} 0,9 \%\end{array}$ & Referenzwerte \\
\hline Kalium $(\mathrm{mmol} / \mathrm{l})$ & 6,5 & 3,9 & $3,5-5,5$ \\
\hline Natrium $(\mathrm{mmol} / \mathrm{l})$ & 109 & 126 & $134-143$ \\
\hline
\end{tabular}

geschrittenen Fällen ist das aufgetriebene Abdomen deutlich erkennbar.

Die Sonografie gibt schnell Aufschluss darüber, ob sich anechogene freie Flüssigkeit im Abdomen befindet ( $\boldsymbol{A} \mathbf{A b} \mathbf{b} . \mathbf{3}$ a und b). Bei Blasenrupturen erscheint die Harnblasenwand relativ dickwandig und die Rupturstelle kann in vielen Fällen dargestellt werden.

Bei labordiagnostischen Untersuchungen fallen betroffene Fohlen durch eine Hyperkaliämie in Verbindung mit einer Hyponatriämie und einer Hypochlorämie auf.

In Zweifelsfällen kann eine Abdominozentese erfolgen, die unter Ultraschallkontrolle am liegenden Fohlen knapp kranial und lateral des Nabels durchzuführen ist. Lässt sich die gewonnene Flüssigkeit makroskopisch und geruchlich nicht eindeutig als Harn identifizieren, hilft eine vergleichende Kreatinin-Bestimmung: Dieses große Molekül reichert sich in der Peritonealhöhle im Vergleich zum Serum etwa um das Doppelte an, da es nicht resorbiert werden kann.

Da es sich beim Uroperitoneum um einen medizinischen Notfall handelt, der chirurgisch versorgt werden muss, gilt es, den Zeitraum bis zur Operation so kurz wie möglich zu halten. Vor allem die Elektrolytverschiebungen (Hyperkaliämie, Hyponatriämie, Hypochlorämie) stellen eine Bedrohung für den Patienten dar [5]. Bei einer bestehenden Hyperkaliämie ist es ratsam, präoperativ mit 0,9\%iger Kochsalzlösung (Richtwert: $20 \mathrm{ml} / \mathrm{kg}$ Körpergewicht als Bolus) zu infundieren, weil es ansonsten zu lebensbedrohlichen Komplikationen (Herzstillstand) bei der Narkoseeinleitung kommen kann ( Tab. 2).

\section{lleus}

Liegt ein Darmverschluss vor, stellt sich das klinisch als akut einsetzende, schnell fortschreitende Kolik dar. In der Folge kommt es zur Dehydrierung, Azidose und Endotoxämie.

Sonografisch lässt sich bei einem Dünndarmileus der atonische, flüssigkeitsgefüllte und hochgradig dilatierte Dünndarm darstellen. In fortgeschrittenen Fäl- 
Tab. 3 Seltenere Ursachen für das Auftreten einer Koliksymptomatik bei neugeborenen Fohlen (Auswahl).

\begin{tabular}{|ll}
\hline Kolikursache & $\begin{array}{l}\text { Diagnostik durch } \\
\text { inkarzerierte Hernien }\end{array}$ \\
\hline Atresia ani/coli/recti & $\begin{array}{l}\text { Palpation, Sonografie, ggf. Probelaparotomie } \\
\text { vorberichtlich fehlender Mekoniumabsatz, } \\
\text { Adspektion (nur Atresia ani), ggf. Sondierung, } \\
\text { Sonografie, Röntgen, Probelaparotomie }\end{array}$ \\
\hline Traumata innerer Organe (z. B. Leber- oder & $\begin{array}{l}\text { klinisches Bild und Labordiagnostik (Anämie), } \\
\text { Sonografie, Abdominozentese, Laparotomie }\end{array}$ \\
\hline Ileokolon-Aganglionose (Lethal-White-foal) & $\begin{array}{l}\text { Vorbericht und entsprechende klinische } \\
\text { Erscheinungen bei weißen Paint-Fohlen }\end{array}$ \\
\hline Peritonitis oder Adhäsionen & $\begin{array}{l}\text { Sonografie } \\
\text { Vorbericht (meist nach Enteritis oder diagnos- } \\
\text { tischen/chirurgischen Eingriffen, aber auch } \\
\text { septisch), Sonografie, Abdominozentese }\end{array}$ \\
postprandiales Auftreten, Überprüfung des Füt- \\
terungsmanagements bei mutterloser Aufzucht
\end{tabular}

len besteht bereits ein Ödem der Darmwand und es findet sich Transsudat in der Bauchhöhle. Bei Dünndarminvaginationen finden sich im sonografischen Querschnitt des betroffenen Darmabschnitts ineinander geschichtete, ödematöse Darmanteile, die an den Anschnitt einer Zwiebel erinnern ( Abb.4). Der Darmabschnitt vor der Stenose ist dilatiert und flüssigkeitsgefüllt.

Die meisten betroffenen Patienten zeigen keine Reaktion auf die Verabreichung von schwachen Analgetika, wie beispielsweise Metamizol, und das Allgemeinbefinden verschlechtert sich schnell. Solange ein mechanischer oder paralytischer Ileus nicht sicher ausgeschlossen werden kann, ist der Einsatz von Butylscopolamin nach Möglichkeit zu vermeiden [6]. Der Einsatz von stärker wirksamen Schmerzmitteln als Metamizol ist für diese Indikation ebenfalls infrage zu stellen, da eine Operation - sofern sie für den Halter des Fohlens infrage kommt - nicht durch Verschleierung der Symptome hinausgezögert werden sollte.

\section{Enteritis/Enterotoxämie}

Hat ein Fohlen Diarrhoe, ist eine begleitende Koliksymptomatik nicht ungewöhnlich. Es kommt allerdings auch vor, dass Fohlen bereits am 1. oder 2. Lebenstag eine bisweilen hochgradige Koliksymptomatik zeigen und außer unterschiedlich dilatierten Dünndarmschlingen in der Sonografie keine klinischen Veränderungen nachweisbar sind. Solche Fohlen entwickeln häufig in den nachfolgenden 12 Stunden eine starke Diarrhoe, die trotz intensivmedizinischer Maßnahmen schwer zu beherrschen ist.

Aufgrund dieser Tatsache ist es ratsam, bei Verdacht auf eine beginnende Enteritis mehrere Kotproben zu asservieren, und direkt anschließend eine breit wirksame intravenöse Antibiotika-Behandlung einzuleiten.

Betroffene Fohlen sind in einer Klinik am besten aufgehoben, auch wenn kein unmittelbares chirurgisches Eingreifen nötig ist. Die Kombination aus Elektrolytverschiebungen, Azidose, Dehydratation und Hypoproteinämie führt dazu, dass eine Infusionstherapie während der Phase der fulminanten Diarrhoe nur flankiert von regelmäßigen labordiagnostischen Kontrollen sinnvoll ist.

Auf den Einsatz von Butylscopolamin sollte auch bei diesen Patienten verzichtet werden, da Motorik und Tonus der Darmmuskulatur durch die Erkrankung bereits herabgesetzt sind. Parasympatholytika können zwar zu einer weiteren Herabsetzung des Tonus führen, dadurch werden allerdings auch die rhythmischen segmentalen Kontraktionen, die propulsive Motorik und die retrograde Peristaltik im Kolon gehemmt. Das bereits bestehende Durchfallgeschehen kann dadurch zusätzlich verstärkt werden [6].

\section{Proktitis}

Diese schmerzhafte Entzündung der Rektumschleimhaut, häufig unter Einbeziehung des Analkegels, erscheint nach den bisher aufgelisteten medizinischen Notfällen etwas deplatziert. Dennoch sollte die Erkrankung Erwähnung finden, da sie häufig bei Fohlen auftritt, bei denen eine Mekoniumobstipation durch mehrfache Spülungen von rektal therapiert wurde oder aber als Begleiterscheinung bei Fohlen mit hochgradiger Diarrhoe.

Vor allem die deutliche Schwellung des Anus, der auch Spannungsrisse aufweisen kann, ist leicht zu erkennen. Der Kotabsatz ist dadurch sehr schmerzhaft, weshalb die Fohlen Tenesmus und auch leichte Koliksymptome zeigen und häufig mit dem Schweif schlagen, und zwar auch dann noch, wenn die Primärerkrankung bereits erfolgreich therapiert ist. Da eine Proktitis 
im Extremfall zum Rektumprolaps führen kann, sollte eine Salbenbehandlung eingeleitet werden. Nach Ausbleiben weiterer Manipulationen oder Sistieren der Diarrhoe klingen die Symptome in aller Regel innerhalb eines kurzen Zeitraums wieder ab.

\section{Atresien (ani/coli/recti)}

Atresien entstehen durch Störungen der embryonalen Entwicklung und treten als angeborene Missbildungen in Erscheinung. Unter diesen ist nur die Atresia ani bei der Erstuntersuchung offensichtlich. Häufiger kommt allerdings die Atresia coli vor ( Abb. 5). Bei Kaltblütern und OveroPaint wird ein autosomal-rezessiver Erbgang vermutet. Neben dem Fehlen des betroffenen Darmabschnittes liegen häufig auch Missbildungen der angrenzenden Darmabschnitte vor, wie beispielsweise neurologische Dysfunktionen oder eine fehlende Tunica muscularis, sodass die Prognose auch bei erfolgreicher Anastomosierung vorsichtig ist. Dennoch wird immer wieder von Einzelfällen berichtet, bei denen eine Operation, wie beispielsweise eine End-zu-Seit-Anastomosierung, zum Erfolg führte [7].

Das Lethal-white-foal-Syndrom (Overolethal-white-Syndrom = OLWS/LWO oder Aganglionose des Ileokolons) ist eine letale Mutation bei Paint und Quarter Horses mit autosomal-rezessivem Erbgang. Daher kommen alle Pferde, die Paint-Blutlinien führen, unabhängig von ihrer tatsächlichen Färbung, als Anlageträger infrage. Seit ein Gentest existiert, ist die Erkrankung selten geworden. Betroffene weiß geborene Fohlen setzen kein Mekonium ab, die Kolikanzeichen beginnen innerhalb von 12 Stunden post natum und führen rasch zum Tode.

\section{Hernien}

Es ist differenzialdiagnostisch zu bedenken, dass Hernien nicht nur an offensichtlichen Lokalisationen wie der Leiste oder am Nabel auftreten können, sondern auch angeborene oder durch Trauma erworbene Zwerchfellhernien bei Fohlen vorkommen können [8]. Allen Hernien ist gemeinsam, dass sie erst bei Inkarzeration zu Kolikerscheinungen führen. Ein chirurgisches Eingreifen ist in diesen Fällen unumgänglich, wobei der Therapieerfolg vom Allgemeinzustand des Fohlens und der Dauer des Krankheitsgeschehens bis zur Operation abhängt. Häufig ist eine Resektion des inkarzerierten Darmabschnitts nötig.

\section{Traumata innerer Organe}

Trat vorberichtlich ein Trauma - am häufigsten ausgelöst durch die Mutterstute auf und kommt es im weiteren Verlauf zur Kolik, ist immer an eine Verletzung innerer Organe zu denken. Rupturen von Leber oder Milz können genauso wie die Verletzung größerer Gefäße zu einem Hämoperitoneum führen, das sich sonografisch gut nachweisen lässt. Im Gegensatz zum Uroperitoneum ist die freie Flüssigkeit nicht anechogen, sondern hypoechogen mit zahlreichen feinen, korpuskulären Reflexen; im weiteren Verlauf oder auch an anderen Stellen des Abdomens kann sich die Blutansammlung durch fortschreitende Organisation auch inhomogen echogen mit reflexreichen Partikeln darstellen [3].

Je nach Lokalisation und Ausmaß des Defekts kann solchen Fohlen geholfen werden, wenn die Blutung lokalisiert und zum Stillstand gebracht werden kann. Häufig sind die entstandenen Schäden aber so groß oder so zahlreich, dass eine Euthanasie unumgänglich ist ( $\bullet$ Abb. 5).

\section{Haemovesica}

Diese seltene postpartale Komplikation führt zu Koliksymptomatik und Tenesmus auf Harn. Sonografisch lässt sich innerhalb der Harnblase ein echogenes Gebilde nachweisen. Dieses Koagel entsteht wahrscheinlich durch eine Einblutung über den Urachus und wurde in früheren Einzelfallberichten erfolgreich chirurgisch entfernt [9], aber auch eine konservative Therapie scheint möglich zu sein [10].

\section{Fazit}

Aufgrund der vielfältigen Ursachen, die eine Kolik beim neugeborenen Fohlen auslösen können, sind Erfahrung und ein vernünftiges Maß an weiterführenden Untersuchungen notwendig, um die richtige Diagnose zu stellen.

Zudem besteht die dringende Notwendigkeit, jedes erkrankte Fohlen nicht nur im
Hinblick auf die akute Koliksymptomatik zu behandeln und zu therapieren. Nur so können mögliche Folgekomplikationen wie beispielsweise Magenulzera, Peritonitis oder Sepsis geringgehalten werden.

\section{Literatur}

1 Sobiraj A, Herfen K, Bostedt H. Die Mekoniumobstipation bei Fohlen: konservative und operative Therapie unter besonderer Berücksichtigung von Komplikationen. Tierärztl. Praxis G 2000; 28, 347-354

2 Ennen S, Wehrend A. Der segmentale Untersuchungsgang für neonatale Fohlen als standardisiertes Verfahren. Prakt. Tierarzt 2010; 91 (3), 222-229

3 Jung C. Sonographische Untersuchung. In: Fey K, Kolm G, Hrsg. Fohlenmedizin. Stuttgart: Enke Verlag 2011: 119-124

4 Behn C, Bostedt H. Technique of abdominal ultrasonography in newborn foals and normal findings. Berl Munch Tierarztl Wochenschr 2000; 113: 335-343

5 Hopster K, Hopster-Iversen C. Diagnose Uroperitoneum - Diagnostik und Therapie von Harnblasenrupturen bei Fohlen. Pferdespiegel 2012; 3: 87-90

6 Richter A, Ungemach FR. Magen-Darm-wirksame Pharmaka. In: Löscher W, Ungemach FR, Kroker R (Hrsg.): Pharmakotherapie bei Haus- und Nutztieren. Stuttgart: Enke Verlag 2014: 251 276

7 Biasutti S, Dart AJ, Dart CM, Uquillas E, Jeffcott LB. End-to-side anastomosis of the left ventral coIon to the small colon in a neonatal foal with segmental agenesis of the large colon. Aust Vet J. 2017; 95(6): 217-219

8 Krohn J, Böhm J, Ennen S, Gerhold HW, Wehrend A. Erfolgreiche chirurgische Therapie einer angeborenen Zwerchfellhernie bei einem vier Tage alten Trakehner-Fohlen. Pferdeheilkunde 2012; 28: 167-170

9 Hospes R, Bostedt $H$. Haemovesica bei einem drei Tage alten Fohlen. Pferdeheilkunde 1998; 14: 205-208

10 Pfundt B, Tietje S. Konservative Therapie einer Haemovesica bei einem Warmblutfohlen: Praktischer Tierarzt 2013; 94 (08): 0706-0711

\section{Online}

https://doi.org/10.1055/s-0043-119168

\section{Dr. Sophia Ennen}

Fachtierärztin für Zuchthygiene und Biotechnologie der Fortpflanzung

Fachtierärztin für kleine Wiederkäuer

Tierarztpraxis Dres. Kraemer

Dorfstraße 104

31637 Rodewald

sophia.ennen@gmx.de 


\section{CVE-Fragebogen \\ Das neugeborene Fohlen als Kolikpatient}

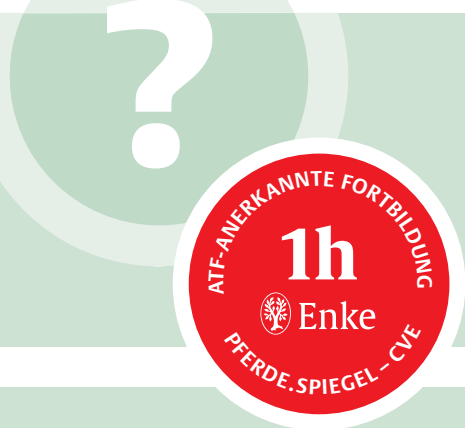

\section{Frage 1}

Eine Kolik in den ersten Lebenstagen eines Fohlens...

a. ... ist immer gastrointestinal bedingt.

b. ... kann durch angeborene Missbildungen ausgelöst sein.

c. ... kann stets konservativ therapiert werden.

d. ... deutet immer auf eine Darmpechverhaltung hin.

e. ... kommt ausschließlich bei prämaturen Fohlen vor.

\section{Frage 2}

Bei welcher der genannten Kolikursachen ist die Diagnose allein durch die Adspektion möglich?
a. Uroperitoneum
b. Mekoniumobstipation
c. Enteritis
d. Ileus
e. Atresia ani

\section{Frage 3}

\section{Eine Darmpechverhaltung...}

a. ... tritt nur bei Hengstfohlen auf.

b. ... ist ausschließlich am 1. Lebenstag zu beobachten.

c. ... kann durch digitale rektale Untersuchung stets zweifelsfrei diagnostiziert werden.

d. ... tritt häufig vergesellschaftet mit Trinkschwäche oder mangelhafter Kolostrumaufnahme auf.

e. ... kann sicher ausgeschlossen werden, wenn der Tierhalter den Abgang einzelner Mekoniumballen beobachtet hat.

\section{Frage 4}

Die begleitenden Elektrolytverschiebungen sind für Fohlen mit Uroperitoneum bedrohlich. Um welche handelt es sich?

a. Hypokaliämie, Hypernatriämie und Hyperchlorämie

b. Hyperkaliämie, Hyponatriämie und Hypochlorämie

c. Hypokaliämie, Hyponatriämie und Hyperchlorämie

d. Hyperkaliämie, Hyperkalzämie und Hypochlorämie

e. Hypokaliämie, Hypokalzämie und Hyperchlorämie

\section{Frage 5}

Welche Aussage trifft auf die Proktitis zu?

a. Sie tritt häufig als alleinige Kolikursache auf.

b. Die Therapie ist aufwändig und langwierig.

c. Sie ist eine häufige Begleiterscheinung bei Mekoniumobstipationen und Enteritiden.

d. Zur Diagnose ist eine Blutuntersuchung notwendig.

e. Ihr Vorliegen beeinträchtigt den Kotabsatz nicht.

\section{Frage 6}

Ab welchem Alter kann eine Enteritis bei Fohlen vorkommen?
a. ab dem 1. Lebenstag
b. ab dem 2. Lebenstag
c. ab dem 3. Lebenstag
d. ab dem 4. Lebenstag
e. ab der 2. Lebenswoche

\section{Frage 7}

Atresien des Darmes...

a. ... zeigen sich am häufigsten als Atresia ani.

b. ... sind bei der klinischen Allgemeinuntersuchung offensichtlich. c. ... haben nach Anastomosierung grundsätzlich eine gute Prognose.

d. ... entstehen durch Störungen während der embryonalen Entwicklung.

e. ... unterliegen einem dominanten Erbgang, sodass Elterntiere von der Zucht auszuschließen sind.

\section{Frage 8}

Welche Maßnahme ist bei einem Fohlen mit Enteritis verzichtbar?

a. Eine Blutuntersuchung einschließlich der Elektrolytwerte.

b. Die Entnahme von Kotproben.

c. Der Einsatz einer systemischen Antibiose.

d. Die Anwendung von Parasympatholytika.

e. Eine Infusionstherapie.

\section{Frage 9}

Welche Maßnahme sollte - unabhängig von der Kolikursache - bei Fohlen durchgeführt werden, die in den ersten Lebenstagen erkrankt sind?

a. Eine Haaranalyse zur Bestimmung von Spurenelementen.

b. Eine Bestimmung des IgG-Gehalts im Blut.

c. Eine endoskopische Untersuchung des Abdomens.

d. Eine Harnanalyse.

e. Eine Lahmheitsuntersuchung.

\section{Frage 10}

Ab welcher sonografisch ermittelten Darmwanddicke ist von entzündlichen oder ödematösen Veränderungen des Darmes auszugehen?
a. $2 \mathrm{~mm}$
b. $3 \mathrm{~mm}$
c. $4 \mathrm{~mm}$
d. $5 \mathrm{~mm}$
e. $6 \mathrm{~mm}$ 


\section{Das neugeborene Fohlen als Kolikpatient}

Sophia Ennen

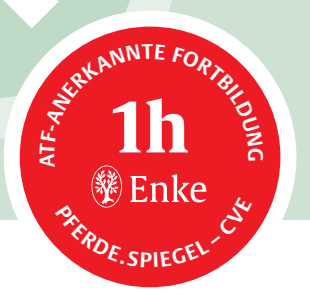

\section{A Lernerfolgskontrolle}

Bitte kreuzen Sie die richtigen

Antworten an! Es ist jeweils nur

1 Antwort pro Frage richtig!

\begin{tabular}{l|l|l|l|l|l|l|} 
Frage 1 & a & b & c & d & e \\
\hline Frage 2 & a & b & c & d & e \\
\hline Frage 3 & a & b & c & d & e \\
\hline Frage 4 & a & b & c & d & e \\
\hline Frage 5 & a & b & c & d & e \\
\hline
\end{tabular}

\begin{tabular}{l|l|l|l|l|l|l|} 
Frage 6 & a & b & c & d & e \\
\hline Frage 7 & a & b & c & d & e \\
\hline Frage 8 & a & b & c & d & e \\
\hline Frage 9 & a & b & c & d & e \\
\hline Frage 10 & a & b & c & d & e
\end{tabular}

\section{B Teilnehmer}

Titel | Name | Vorname

Straße | Hausnummer

PLZ | Ort

Beruf

\section{Ihr Ergebnis wird vom Verlag ausgefüllt}

Sie haben von Fragen

$\square$ bestanden und 1 ATF-Stunde erhalten

$\square$ nicht bestanden.

richtig beantwortet und somit

Stuttgart, den

Stempel | Unterschrift

\section{Teilnahmebedingungen für Abonnenten des pferde.spiegel kostenlos}

Für diese Fortbildung können Sie 1 ATF-Fortbildungsstunde anerkannt bekommen. Hierfür - müssen mindestens $70 \%$ der Fragen richtig beantwortet sein.

- muss der Antwortbogen vollständig ausgefüllt sein. Unvollständig ausgefüllte Bögen können nicht berücksichtigt werden.

\section{E Erklärung}

Ich versichere, dass ich die Beantwortung der Fragen selbst und ohne fremde Hilfe durchgeführt habe.
- muss im markierten Feld* Ihre Abonnentennummer eingetragen oder eine pferde. spiegel-CVE-Wertmarke aufgeklebt sein.

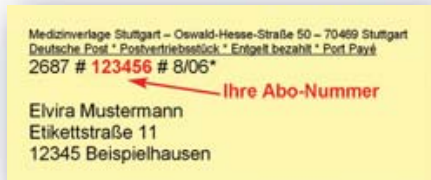

pferde.spiegel-CVE-Wertmarken für Nichtabonnenten können beim Verlag zu folgenden Bedingungen erworben werden: 4er-Pack Wertmarken, Preis 49,95€ inkl. MwSt., Artikel-Nr. 903001. Bitte richten Sie die Bestellungen an: Georg Thieme Verlag KG,

KundenServiceCenter Buch, Postfach 301120, 70451 Stuttgart.

* Nichtabonnenten bitte hier pferde. spiegel-CVE-Wertmarke aufkleben. Abonnenten bitte Abonnentennummer eintragen: 